\title{
Фармакоэкономическое исследование
}

\section{применения магнитно-резонансной томографии с гадоксетовой кислотой в дополнение к компьютерной томографии с контрастированием при диагностике гепатоцеллюлярного рака}

\author{
Кармазановский Г.Г.1, 2 , Волобуева А.А. ${ }^{3}$ \\ ${ }^{1}$ ФГБУ «Национальный медицинский исследовательский центр хирургии им. А.В. Вишневского» Минздрава России, \\ Большая Серпуховская ул., 27, Москва, 117997, Российская Федерация \\ 2 ФГАОУ ВО «Российский национальный исследовательский медицинский университет им. Н.И. Пирогова» \\ Минздрава России, ул. Островитянова, 1, Москва, 117997, Российская Федерация \\ ${ }^{3}$ АО «Байер», \\ 3-я Рыбинская ул., 18, стр. 2, Москва, 107113, Российская Федерация
}

\begin{abstract}
Кармазановский Григорий Григорьевич, д. М. Н., профессор, чл.-корр. РАН, заведующий отделом лучевых методов диагностики и лечения ФГБУ «Национальный медицинский исследовательский центр хирургии им. А.В. Вишневского» Минздрава России; профессор кафедры лучевой диагностики и терапии ФГАОУ ВО «Российский национальный исследовательский медицинский университет им. Н.И. Пирогова» Минздрава России; https://orcid.org/0000-0002-9357-0998
\end{abstract}

Волобуева Арина Александровна, специалист по фармакоэкономике отдела доступа на рынок и ценообразования АО «Байер»; https://orcid.org/0000-0002-1824-8712

\begin{abstract}
Резюме
Цель: провести оценку фармакоэкономических преимуществ применения магнитно-резонансной томографии (МРТ) с гепатоспецифическим контрастным средством - гадоксетовой кислотой в дополнение к компьютерной томографии (КТ) с контрастированием при диагностике гепатоцеллюлярного рака (ГЦР). Материал и методы. Проведено моделирование, в ходе которого получены результаты фармакоэкономического исследования: анализа эффективности, анализа затрат и анализа «затраты-эффективность». При оценке затрат были учтены только прямые медицинские затраты (затраты на КТ с контрастированием, стоимость магнитно-резонансного контрастного средства (МРКС) и стоимость лечения, выполненного пациенту с установленным диагнозом ГЦР после диагностического исследования). Финансирование осуществлялось в рамках системы обязательного медицинского страхования. Расчет результатов приведен на 1 пациента.

Результаты. Анализ эффективности показал, что схема диагностики КТ + МРТ с гадоксетовой кислотой является наиболее эффективным комплексом исследований для диагностики ГЦР, поскольку ее применение позволило достичь почти у 50\% пациентов практически 5-летней общей выживаемости (4,8 года), что статистически значимо выше, чем при диагностике ГЦР с помощью КТ + МРТ с внеклеточными гадолинийсодержащими МРКС (2,3 года) или КТ (3,7 года). Анализ «затраты-эффективность» продемонстрировал, что схема диагностики с гадоксетовой кислотой является доминантной: на достижение 1 года жизни при ее использовании требуется меньшее количество затрат (14 862 руб.) в сравнении с КТ + МРТ с внеклеточными гадолинийсодержащими МРКС (25 293 руб.) или КТ (46 540 руб.). Согласно результатам расчета инкрементального показателя «затраты-эффективность» применение комплекса исследований с гадоксетовой кислотой характеризуется наименьшими дополнительными затратами на достижение 1 дополнительного года жизни.

Заключение. В результате проведенного фармакоэкономического исследования установлено, что использование МРТ с гепатоспецифическим контрастным средством - гадоксетовой кислотой в дополнение к КТ с контрастированием является эффективным и экономически выгодным способом ранней диагностики ГЦР.

Ключевые слова: гепатоцеллюлярный рак; гепатоцеллюлярная карцинома; магнитно-резонансные контрастные средства; гепатоспецифическое контрастное средство; гадоксетовая кислота; внеклеточные гадолинийсодержащие контрастные средства; гадобеновая кислота; гадодиамид; гадопентетовая кислота; гадотеровая кислота; гадобутрол; компьютерная томография; магнитно-резонансная томография; анализ эффективности; анализ затрат; анализ «затраты-эффективность».

Конфликт интересов. Г.Г. Кармазановский сотрудничает с АО «Байер», А.А. Волобуева является сотрудником АО «Байер». АО «Байер» не оказывало какого-либо влияния на дизайн исследования, анализ и интерпретацию результатов.
\end{abstract}


Финансирование. Данное фармакоэкономическое исследование выполнено при поддержке АО «Байер». Для цитирования: Кармазановский Г.Г., Волобуева А.А. Фармакоэкономическое исследование применения магнитно-резонансной томографии с гадоксетовой кислотой в дополнение к компьютерной томографии с контрастированием при диагностике гепатоцеллюлярного рака. Вестник рентгенологии и радиологии. 2021; 102(5): 284-95. https://doi.org/10.20862/0042-4676-2021-102-5-284-295

Для корреспонденции: Кармазановский Григорий Григорьевич, E-mail: karmazanovsky@ixv.ru

\title{
Pharmacoeconomic Study on the Application of Magnetic Resonance Imaging with Gadoxetic Acid in Addition to Contrast-Enhanced Computed Tomography in Diagnosis of Hepatocellular Carcinoma
}

\section{Grigory G. Karmazanovsky ${ }^{1,2}$, Arina A. Volobueva ${ }^{3}$}

\author{
${ }^{1}$ Vishnevsky National Medical Research Center of Surgery, Ministry of Health of the Russian Federation, \\ ul. Bolshaya Serpukhovskaya, 27, Moscow, 117997, Russian Federation \\ 2 Pirogov Russian National Research Medical University, Ministry of Health of the Russian Federation, \\ ul. Ostrovityanova, 1, Moscow, 117997, Russian Federation \\ ${ }^{3}$ Bayer JSC, \\ ul. Tretya Rybinskaya, 18, str. 2, Moscow, 107113, Russian Federation
}

Grigory G. Karmazanovsky, Dr. Med. Sc., Professor, Corresponding Member of RAS, Head of Radiology Department, Vishnevsky National Medical Research Center of Surgery; Professor, Chair of Radiology, Pirogov Russian National Research Medical University; https://orcid.org/0000-0002-9357-0998

Arina A. Volobueva, Health Economics Specialist, Market Access and Pricing Department, Bayer JSC; https://orcid.org/0000-0002-1824-8712

\begin{abstract}
Objective: to evaluate pharmacoeconomic advantages of the application of magnetic resonance imaging (MRI) with hepatobiliary-specific contrast agent - gadoxetic acid in addition to contrast-enhanced computed tomography (CT) in the diagnosis of hepatocellular carcinoma (HCC).

Material and methods. We performed the modeling and obtained the results of the pharmacoeconomic study: effectiveness analysis, cost analysis, and cost-effectiveness analysis. The cost analysis included only direct medical costs (the cost of contrast-enhanced CT, gadolinium-based contrast agent (GBCA) for MRI, and therapy per a patient with verified HCC diagnosis after diagnostic procedures). The financing was performed from the Compulsary Health Insurance Fund. The calculations were made per 1 patient.

Results. The effectiveness analysis showed that the diagnostic scheme CT + MRI with gadoxetic acid was the most effective complex for the diagnosis of HCC because its application provided $50 \%$ of patients with 5 -year overall survival (4.8 years), which was significantly higher than in patients who were diagnosed HCC with CT + MRI with extracellular GBCA (2.3 years) or CT (3.7 years). The cost-effectiveness analysis showed that the diagnostic scheme with gadoxetic acid was dominant because each disability-adjusted life year required fewer costs (14 862 rubles) in comparison with CT + MRI with extracellular GBCA (25 293 rubles) or CT (46 540 rubles). According to the results of the incremental cost-effectiveness ratio analysis, the application of the diagnostic complex with gadoxetic acid was characterized by the lowest rate of additional costs per 1 disability-adjusted life year.

Conclusion. The pharmacoeconomic study showed that the application of MRI with a hepatospecific contrasting agent - gadoxetic acid in addition to contrast-enhanced CT was an effective and economically beneficial method of early HCC diagnosis.

Keywords: hepatocellular carcinoma; magnetic resonance contrast agents; hepatobiliary-specific contrast agent; gadoxetic acid; extracellular gadolinium-based contrast agents; gadobenic acid; gadodiamide; gadopentetic acid; gadoteric acid; gadobutrol; computed tomography; magnetic resonance imaging; effectiveness analysis; cost analysis; cost-effectiveness analysis.

Conflict of interest. Grigory G. Karmazanovksy cooperates with Bayer JSC. Arina A. Volobueva is an employee of Bayer JSC. Bayer JSC did not influence in any way the study design, analysis, or interpretation of the results.
\end{abstract}


For citation: Karmazanovsky GG, Volobueva AA. Pharmacoeconomic study on the application of magnetic resonance imaging with gadoxetic acid in addition to contrast-enhanced computed tomography in diagnosis of hepatocellular carcinoma. Journal of Radiology and Nuclear Medicine. 2021; 102(5): $284-95$ (in Russian). https://doi.org/10.20862/0042-4676-2021-102-5-284-295

For corresponding: Grigory G. Karmazanovsky, E-mail: karmazanovsky@ixv.ru

Received July 19, $2021 \quad$ Revised September 24, $2021 \quad$ Accepted September 25, 2021

\section{Введение}

Гепатоцеллюлярный рак (ГЦР) является одной из первичных злокачественных опухолей печени. Уникальность этого новообразования в том, что при наличии общих исходных нарушений в гепатоците (опухолевая трансформация) существует три известных на данный момент механизма развития опухоли, а значит, и ее первичных проявлений при лучевой диагностике, влияющих на выбор тактики лечения $[1,2]$.

Первый тип опухоли - это ГЦР на фоне хронического воспалительного процесса, гепатита, при нормальной паренхиме печени. Как и другие типы, чаще всего он выявляется случайно, если опухоль не достигла больших размеров и не успела существенно повлиять на метаболизм (онкомаркеры, снижение веса). В этих случаях важнейшую роль играют критерии дифференциальной диагностики при томографических исследованиях с использованием контрастного усиления [3, 4].

По мере роста опухоли и вовлечения структур внутри печени и за ее пределами неминуемо возникает вопрос о возможности хирургического вмешательства или его сочетания с химиотерапией на дооперационном этапе и в послеоперационном периоде. Критерии резектабельности и операбельности также оценивают, опираясь на данные томографических исследований [5].

Второй тип - фиброламеллярный ГЦР, который является особой формой данной опухоли, чаще выявляемой у молодых. При первичной диагностике он, как правило, огромных размеров $[2,6]$.

Третий тип - ГЦР на фоне цирроза печени, который требует дифференциации четырех типов узловых образований на фоне цирротической трансформации паренхимы печени: узлов регенерации печеночной ткани, диспластических узлов без макропроявлений злокачественности, диспластических узлов с макропроявлениями злокачественности, когда видны так называемые узлы в узле. Узел в печени может быть одной из стадий развития ГЦР [7, 8]. Важнейшую роль в прогнозе играют оценка капсулы опухоли и степень инвазии сосудов. В большинстве случаев современные лучевые исследования отвечают и на эти вопросы $[9,10]$.

В настоящее время лучевая неинвазивная диагностика продвинулась столь далеко, что благодаря применению магнитно-резонансной томо- графии (МРТ) с гепатоспецифическим магнитнорезонансным контрастным средством (МРКС) В комбинации с диффузионно-взвешенными МРТ-изображениями можно дифференцировать разные типы узловых образований печени и даже стадию ГЦР [3, 9-11]. Возможностей много, но их большое количество не означает их автоматическое использование. Все ли они реализуются в полной мере в каждом случае при обследовании и планировании лечения конкретного пациента?

Вырабатываются определенные диагностиколечебные стратегии, публикуются клинические рекомендации. Здравоохранение, наконец, считает затраты, оценивая целесообразность тех или иных программ обследования и лечения. Однако то, что лежит на поверхности, не всегда отражает объективную реальность. Психологически трудно свыкнуться с мыслью, что то, что считается «дорогим", может быть более целесообразным при достижении конечного результата, чем очевидное «дешевое».

В международных рекомендациях по диагностике заболеваний печени МРТ с гепатоспецифическим МРКС, гадоксетовой кислотой, включена в первую линию диагностической визуализации ГЦР. В российских руководствах метод также рекомендован, но не занимает первенство среди диагностических исследований [3-5, 12-19].

Данная работа была выполнена с целью показать с помощью фармакоэкономического исследования объективную реальность при современной диагностике ГЦР, основанной на МРТ с гадоксетовой кислотой.

Опубликованные фармакоэкономические исследования применения МРТ с гадоксетовой кислотой, МРТ с внеклеточными контрастными средствами (в т.ч. гадолинийсодержащими) и КТ с контрастированием показали, что МРТ с гадоксетовой кислотой обладает самой высокой диагностической эффективностью и сводит к минимуму необходимость в дополнительных подтверждающих исследованиях, что приводит к уменьшению затрат на диагностику [20-22].

В условиях здравоохранения Российской Федерации фармакоэкономическое исследование применения контрастных средств при диагностике ГЦР ранее не проводилось, поэтому данная работа является актуальной. 


\section{Материал и методы}

При построении модели были использованы такие методы фармакоэкономического исследования, как анализ эффективности, анализ затрат и анализ «затраты-эффективность». Моделирование было проведено в программном пакете Microsoft Excel версии 2008 г. (сборка 13127.21624).

\section{Анализ эффективности}

На первом этапе фармакоэкономического исследования применения контрастных средств при диагностике ГЦР было проведено изучение опубликованных результатов клинических исследований (в т.ч. рандомизированных), метаанализов и систематических обзоров, посвященных применению гадоксетовой кислоты при диагностике ГЦР, за последние 10 лет. Информационный поиск осуществлялся в базе данных PubMed согласно следующей стратегии: «(gadoxetic acid) AND (gadoxetic acid-enhanced) AND ((MRI) OR (magnetic resonance imaging) OR (MR imaging)) AND (hepatocellular carcinoma) AND (((computed tomography) OR (CT) OR (MDCT)) OR ((extracellular contrast) OR (extracellular media-enhanced)))». При этом было найдено 84 публикации, из них 32 являются полнотекстовыми и находятся в свободном доступе. Было выявлено, что в 7 из 32 публикаций не проводилось сравнение МРТ с гадоксетовой кислотой и другими методами диагностики ГЦР. В 24 работах оценены лишь визуальные характеристики (диагностическая точность и чувствительность) МРТ с гадоксетовой кислотой и КТ и/или МРТ с внеклеточными гадолинийсодержащими МРКС.

Только в одной публикации по результатам ретроспективного когортного исследования T.W. Kang et al. (2020 г.), в котором изучалось применение гадоксетовой кислоты при обследовании более чем 30 тыс. больных ГЦР в Южной Корее, представлено прямое сравнение эффективности (выживаемости пациентов после диагностики ГЦР) применения гадоксетовой кислоты и других гадолинийсодержащих контрастных средств [23]. Основная цель исследования заключалась в оценке эффективности дополнительного использования МРТ с гадоксетовой кислотой в диагностике больных ГЦР, которым ранее была проведена КТ. Согласно протоколу исследования пациенты были разделены на три группы: в 1-й группе была проведена только КТ, во 2-й и 3-й группах дополнительно к КТ выполняли МРТ с внеклеточными гадолинийсодержащими МРКС или с гадоксетовой кислотой. После установления диагноза участникам исследования было проведено определенное лечение: радиочастотная абляция, трансартериальная химиоэмболизация, таргетная или лучевая терапия. Некоторым пациентам лечение не проводилось. Горизонт наблюдения за больными составил 7 лет (с 1 января 2008 г. по 31 декабря 2014 г.) [23].

Первичная конечная точка исследования T.W. Kang et al. включала смерть от всех причин, вторичная - смерть от рака печени. Общую выживаемость пациентов рассчитывали от момента постановки диагноза ГЦР до смерти или окончания исследования. Для оценки показателей отношения рисков смерти от всех причин и от рака печени использовали регрессионную модель пропорциональных рисков Кокса с 95\% доверительным интервалом (ДИ). Исследование пропорциональных рисков Кокса осуществляли с помощью графиков логарифмической функции выживаемости и графиков остатков Шенфельда. При этом пороговый уровень статистической значимости (р-значение) составил 0,05. В исследовании также был проведен многофакторный анализ, в котором для сопоставления результатов в трех группах пациентов была проведена корректировка по таким критериям, как пол и возраст, локализация опухоли, год постановки диагноза, причина заболевания печени, индекс Чарлсона, процентиль дохода, район проживания и назначенное после диагностики лечение [23].

Фактические данные этой публикации легли в основу расчетов экономической эффективности, проведенных в настоящей работе. В соответствии с исследованием T.W. Kang et al. [23] при выполнении фармакоэкономического исследования для сравнения мы использовали три схемы диагностики ГЦР:

1) КТ - мультифазная компьютерная томография с контрастированием;

2) KT + MPT c Gd - мультифазная компьютерная томография с контрастированием в сочетании с магнитно-резонансной томографией с усилением внеклеточными гадолинийсодержащими контрастными средствами;

3) КТ + МРТ с гадоксетовой кислотой - мультифазная компьютерная томография с контрастированием в сочетании с магнитно-резонансной томографией с гадоксетовой кислотой.

В качестве контрастных средств, применяемых для диагностики ГЦР с помощью КТ + МРТ с Gd, были использованы гадобеновая кислота, гадодиамид, гадопентетовая кислота, гадотеровая кислота, гадобутрол, а также комбинации представленных контрастных средств. В комплексе диагностических исследований КТ + МРТ с гадоксетовой кислотой применялась гадоксетовая кислота [23].

\section{Анализ затрат}

Следующим этапом проводили анализ затрат. Были рассчитаны прямые затраты, которые включали в себя затраты на КТ с контрастированием, MPKC и лечение, назначенное пациенту с установленным диагнозом ГЦР после диагностического исследования. 
Распределение контрастных средств для магнитно-резонансной томографии среди пациентов, которым была проведена диагностика гепатоцеллюлярного рака

Table 1

The distribution of contrast agents for magnetic resonance imaging among patients who underwent hepatocellular carcinoma diagnosis

\begin{tabular}{|c|c|c|}
\hline $\begin{array}{l}\text { Метод диагностики / } \\
\text { Diagnostic method }\end{array}$ & $\begin{array}{l}\text { Международное непатентованное наименование / } \\
\text { International nonproprietary name }\end{array}$ & $\begin{array}{l}\text { Распределение, \% / } \\
\text { Distribution, \% }\end{array}$ \\
\hline \multirow{15}{*}{$\begin{array}{l}\mathrm{KT}+\mathrm{MPT} \text { c Gd / CT + MRI } \\
\text { with GBCA }\end{array}$} & Гадобеновая кислота / Gadobenic acid & 28,07 \\
\hline & Гадодиамид / Gadodiamide & 26,94 \\
\hline & Гадопентетовая кислота / Gadopentetic acid & 23,70 \\
\hline & Гадотеровая кислота / Gadoteric acid & 4,44 \\
\hline & Гадобутрол / Gadobutrol & 15,63 \\
\hline & Гадобеновая кислота + гадодиамид / Gadobenic acid + gadodiamide & 0,16 \\
\hline & $\begin{array}{l}\text { Гадобеновая кислота + гадопентетовая кислота / Gadobenic acid + } \\
\text { gadopentetic acid }\end{array}$ & 0,04 \\
\hline & Гадобеновая кислота + гадобутрол / Gadobenic acid + gadobutrol & 0,04 \\
\hline & $\begin{array}{l}\text { Гадобеновая кислота + гадотеровая кислота / Gadobenic acid + } \\
\text { + gadoteric acid }\end{array}$ & 0,27 \\
\hline & Гадодиамид + гадопентетовая кислота / Gadodiamide + gadopentetic acid & 0,16 \\
\hline & Гадодиамид + гадобутрол / Gadodiamide + gadobutrol & 0,08 \\
\hline & Гадодиамид + гадотеровая кислота / Gadodiamide + gadoteric acid & 0,12 \\
\hline & Гадопентетовая кислота + гадобутрол / Gadopentetic acid + gadobutrol & 0,23 \\
\hline & $\begin{array}{l}\text { Гадопентетовая кислота + гадотеровая кислота / Gadopentetic acid + } \\
\text { + gadoteric acid }\end{array}$ & 0,08 \\
\hline & Гадобутрол + гадотеровая кислота / Gadobutrol + gadoteric acid & 0,04 \\
\hline $\begin{array}{l}\text { KT + MPT с гадоксетовой } \\
\text { кислотой / СТ + MRI with } \\
\text { gadoxetic acid }\end{array}$ & Гадоксетовая кислота / Gadoxetic acid & 100,00 \\
\hline
\end{tabular}

Примечание. КТ - компьютерная томография; МРT - магнитно-резонансная томография; Gd - внеклеточные гадолинийсодержащие контрастные средства.

Note. CT - computed tomography; MRI - magnetic resonance imaging; GBCA - extracellular gadolinium-based contrast agents.

Первая составляющая анализа затрат - стоимость КТ с контрастированием. Для ее расчета было взято среднее значение стоимости тарифа КТ органов забрюшинного пространства и/или брюшной полости и забрюшинного пространства с внутривенным болюсным контрастированием или другого наиболее подходящего тарифа (ввиду отсутствия в некоторых субъектах указанных ранее тарифов) из тарифных соглашений по программе обязательного медицинского страхования (ОМC) на территории субъектов РФ $\Phi^{1}$. Таким образом, средняя стоимость КТ с контрастированием составила 7027 руб.

\footnotetext{
1 Тарифные соглашения по программе обязательного медицинского страхования на территории субъектов РФ.
}

Второй составляющей анализа затрат являлась стоимость контрастного средства для МРТ c Gd и MPT с гадоксетовой кислотой. Ее расчет осуществляли на основе стоимости одного введения МРКС и данных по распределению используемых контрастных средств или их комбинаций среди больных ГЦР [23] (табл. 1, 2). В связи с отсутствием гадотеровой кислоты в перечне жизненно необходимых и важнейших лекарственных препаратов в Российской Федерации, а следовательно, и отсутствием зарегистрированной предельной отпускной цены производителя, стоимость одного введения МРКС была определена на основе средневзвешенных тендерных цен за упаковку (за период с 1 июля 2020 г. по 1 июля 2021 г.) $)^{2}$.

\footnotetext{
2 IQVIA (бывш. IMS) «Тендеры ГЛС и БАД в РФ».
} 
Weighted average bid prices for contrast agents for magnetic resonance imaging

\begin{tabular}{|c|c|c|c|}
\hline $\begin{array}{c}\text { Международное непатентованное } \\
\text { наименование / International } \\
\text { nonproprietary name }\end{array}$ & $\begin{array}{c}\text { Торговое наименование / } \\
\text { Trade name }\end{array}$ & $\begin{array}{l}\text { Дозировка, упаковка / } \\
\text { Dosage, packaging }\end{array}$ & $\begin{array}{l}\text { Цена за упаковку, руб./ } \\
\text { Price per package, rub. }\end{array}$ \\
\hline $\begin{array}{l}\text { Гадобеновая кислота / } \\
\text { Gadobenic acid }\end{array}$ & Мультихэнс ${ }^{\circledR}$ / MultiHance ${ }^{\circledR}$ & $\begin{array}{l}10 \text { мл № } 1 / \\
10 \text { ml №. } 1\end{array}$ & 2280,27 \\
\hline \multirow[t]{2}{*}{$\begin{array}{l}\text { Гадодиамид / } \\
\text { Gadodiamide }\end{array}$} & $\begin{array}{l}\text { Гадодиамид Джодас }{ }^{\circledR} / \\
\text { Jodas Gadodiamide }\end{array}$ & $\begin{array}{l}15 \text { мл № } 10 / \\
15 \text { ml №. } 10\end{array}$ & 10213,32 \\
\hline & Омнискан ${ }^{\circledR} /$ Omniscan ${ }^{\circledR}$ & $\begin{array}{l}15 \text { мл № } 10 / \\
15 \text { ml №. } 10\end{array}$ & 16795,54 \\
\hline \multirow[t]{2}{*}{$\begin{array}{l}\text { Гадопентетовая кислота / } \\
\text { Gadopentetic acid }\end{array}$} & $\begin{array}{l}\text { Гадопентетовая кислота Джодас }{ }^{\circledR} / \\
\text { Jodas Gadopentetic Acid® }\end{array}$ & $\begin{array}{l}15 \text { мл № } 10 / \\
15 \text { ml №. } 10\end{array}$ & 11712,75 \\
\hline & Магневист ${ }^{\circledR}$ / Magnevist ${ }^{\circledR}$ & $\begin{array}{l}15 \text { мл № } 10 \text { / } \\
15 \text { ml №. } 10\end{array}$ & 19745,36 \\
\hline \multirow[t]{3}{*}{$\begin{array}{l}\text { Гадотеровая кислота / } \\
\text { Gadoteric acid }\end{array}$} & Дотарем ${ }^{\circledR} /$ Dotarem ${ }^{\circledR}$ & $\begin{array}{l}15 \text { мл № } 1 / \\
15 \text { ml №.1 }\end{array}$ & 3025,38 \\
\hline & Кларискан ${ }^{\circledR} /$ Clariscan $^{\circledR}$ & $\begin{array}{l}15 \text { мл № } 10 / \\
15 \text { ml №. } 10\end{array}$ & 19420,84 \\
\hline & $\begin{array}{l}\text { Гадотеровая кислота Джодас }{ }^{\circledR} / \\
\text { Jodas Gadoteric Acid® }\end{array}$ & $\begin{array}{l}20 \text { мл № } 1 / \\
20 \text { ml No.1 }\end{array}$ & 4527,60 \\
\hline \multirow[t]{3}{*}{$\begin{array}{l}\text { Гадобутрол / } \\
\text { Gadobutrol }\end{array}$} & Гадовист ${ }^{\circledR}$ / Gadovist ${ }^{\circledR}$ & $\begin{array}{l}7,5 \text { мл № } 5 \text { / } \\
7,5 \mathrm{ml} \text { №. } 5\end{array}$ & 14842,06 \\
\hline & $\begin{array}{l}\text { Гадовист Полисан }{ }^{\circledR} / \\
\text { Gadovist Polysan }^{\circledR}\end{array}$ & $\begin{array}{l}15 \text { мл № } 5 \text { / } \\
15 \text { ml №. } 5\end{array}$ & 28105,12 \\
\hline & Гадобусканн ${ }^{\circledR}$ / Gadobuscan® ${ }^{\circledR}$ & $\begin{array}{l}15 \text { мл № } 5 \text { / } \\
15 \text { ml №. } 5\end{array}$ & 9264,99 \\
\hline $\begin{array}{l}\text { Гадоксетовая кислота / } \\
\text { Gadoxetic acid }\end{array}$ & Примовист ${ }^{\circledR}$ / Primovist $^{\circledR}$ & $\begin{array}{l}10 \text { мл № 1/ } \\
10 \text { ml №. } 1\end{array}$ & 7839,91 \\
\hline
\end{tabular}

Итоговая стоимость МPKC с Gd/гадоксетовой кислотой была рассчитана по следующей формуле:

$$
\mathrm{C}_{\mathrm{MPKC}}=\sum_{i=1}^{n} \mathrm{C}_{i} \times Д_{i},
$$

где $\mathrm{C}_{i}$ - стоимость одного введения $i$-го МРКС или их комбинации (руб.); Дi - доля пациентов, которым вводилось $i$-е МРКС или их комбинации (\%).

Для расчета третьей составляющей анализа затрат, а именно средней стоимости лечения 1 пациента, использовали данные о распределении больных по назначенному лечению после проведения диагностики и установления диагноза ГЦР [23] (табл. 3). Все расчеты осуществляли в соответствии с методическими рекомендациями по способам оплаты медицинской помощи за счет средств ОМС на 2021 r. $^{3}$, а также нормативами финансовых

\footnotetext{
3 Письмо Министерства здравоохранения РФ и Федерального фонда обязательного медицинского страхования от 30 декабря 2020 г. №№ 11-7/и/2-20691, 00-10-26-2-04/11-51 «О Методических рекомендациях по способам оплаты медицинской помощи за счет средств обязательного медицинского страхования».
}

затрат, предусмотренными Программой государственных гарантий бесплатного оказания гражданам медицинской помощи (ПГГ) на $2021 r^{4}$.

Средние нормативы финансовых затрат на 1 случай госпитализации в медицинских организациях, оказывающих медицинскую помощь в условиях дневного (ДС) и круглосуточного (КС) стационаров за счет средств ОМС, представлены в таблице $4^{3}$.

Используемые в расчете стоимости лечения 1 пациента коэффициенты затратоемкости, полученные из справочника клинико-статистических групп (КСГ), указаны в таблицах 5 и $6^{2}$.

Стоимость определенного вида лечения ГЦР в ДС или КС была рассчитана по следующей формуле ${ }^{5}$ :

\footnotetext{
${ }^{4}$ Постановление Правительства РФ от 28 декабря 2020 г. № 2299 «О Программе государственных гарантий бесплатного оказания гражданам медицинской помощи на 2021 год и на плановый период 2022 и 2023 годов».

${ }^{5}$ Приказ ФГБУ «ЦККЭМП» Минздрава России от 29 декабря 2018 г. № 242-од «Методические рекомендации по проведению сравнительной клинико-экономической оценки лекарственного препарата».
} 
Вероятность назначения лечения после проведения диагностики и установления диагноза гепатоцеллюлярного рака, \%

Possibility of indication of treatment after diagnostics and verification of hepatocellular carcinoma diagnosis, \%

\begin{tabular}{|c|c|c|c|}
\hline Вид лечения / Type of treatment & $\mathrm{KT} / \mathrm{CT}$ & $\begin{array}{c}\mathrm{KT}+\mathrm{MPT} \text { c Gd / } \\
\mathrm{CT}+\mathrm{MRI} \text { with GBCA }\end{array}$ & $\begin{array}{c}\text { KT + MPT с гадоксетовой кислотой / } \\
\text { CT + MRI with gadoxetic acid }\end{array}$ \\
\hline Трансплантация печени / Liver transplantation & 0,2 & 0,5 & 0,2 \\
\hline Резекция / Resection & 6,1 & 17,1 & 24,0 \\
\hline Радиочастотная абляция / Radiofrequency ablation & 3,7 & 4,2 & 10,1 \\
\hline $\begin{array}{l}\text { Трансартериальная химиоэмболизация / } \\
\text { Transarterial chemoembolization }\end{array}$ & 39,6 & 36,8 & 35,4 \\
\hline Таргетная терапия / Targeted therapy & 0,5 & 0,3 & 0,5 \\
\hline $\begin{array}{l}\text { Другое лечение (включает лучевую терапию) / } \\
\text { Other (includes radiation therapy) }\end{array}$ & 5,8 & 7,8 & 6,6 \\
\hline Нет лечения / No treatment & 44,2 & 33,2 & 23,3 \\
\hline
\end{tabular}

Средние нормативы финансовых затрат на 1 случай госпитализации

Average norms for financial expenses per one case of hospitalization

\begin{tabular}{cc}
\hline Стационар / Facility & Норматив затрат, руб. / Norm for costs, rub. \\
\hline Круглосуточный / All-day & 22261,50 \\
Дневной / Day-time & 37382,30 \\
\hline
\end{tabular}

Таблица 5

Коэффициенты затратоемкости госпитализаций для лечения гепатоцеллюлярного рака в дневном стационаре

Table 5

Cost intensity ratios of hospitalizations for the treatment of hepatocellular carcinoma in a day-time inpatient facility

\begin{tabular}{|c|c|c|}
\hline № КСГ / CSG No. & Расшифровка КСГ / CSG decoding & $\mathrm{K} 3 / \mathrm{CIR}$ \\
\hline ds19.037 & $\begin{array}{l}\text { Лекарственная терапия при ЗНО (кроме лимфоидной и кроветворной тканей), } \\
\text { взрослые (уровень 1) / Drug therapy for MT (except lymphoid and hematopoietic tissues), } \\
\text { adults (level 1) }\end{array}$ & 0,48 \\
\hline ds19.050 & Лучевая терапия (уровень 1) / Radiation therapy (level 1) & 0,74 \\
\hline ds19.052 & Лучевая терапия (уровень 3) / Radiation therapy (level 3) & 2,22 \\
\hline ds19.055 & Лучевая терапия (уровень 6) / Radiation therapy (level 6) & 3,8 \\
\hline ds19.059 & $\begin{array}{l}\text { Лучевая терапия в сочетании с лекарственной терапией (уровень 2) / } \\
\text { Radiation therapy in combination with drug therapy (level 2) }\end{array}$ & 4,4 \\
\hline ds19.061 & $\begin{array}{l}\text { Лучевая терапия в сочетании с лекарственной терапией (уровень 4) / } \\
\text { Radiation therapy in combination with drug therapy (level 4) }\end{array}$ & 13,27 \\
\hline ds19.062 & $\begin{array}{l}\text { Лучевая терапия в сочетании с лекарственной терапией (уровень 5) / } \\
\text { Radiation therapy in combination with drug therapy (level 5) }\end{array}$ & 25,33 \\
\hline
\end{tabular}

Примечание. КСГ - клинико-статистическая группа; КЗ - коэффициент затратоемкости; ЗНО - злокачественные новообразования.

Note. CSG - clinical and statistical group; CIR - cost intensity ratio; MT - malignant tumors. 
Input intensity coefficients of hospitalizations for the treatment of hepatocellular carcinoma in an all-day inpatient facility

\begin{tabular}{|c|c|c|}
\hline № КСГ / CSG No. & Расшифровка КСГ / CSG decoding & $\mathrm{K} 3 / \mathrm{CIR}$ \\
\hline st32.006 & $\begin{array}{l}\text { Операции на печени и поджелудочной железе (уровень 2) / } \\
\text { Biliary and pancreatic surgery (level 2) }\end{array}$ & 2,69 \\
\hline st19.062 & $\begin{array}{l}\text { Лекарственная терапия при ЗНО (кроме лимфоидной и кроветворной тканей), взрослые } \\
\text { (уровень 1) / Drug therapy for MT (except lymphoid and hematopoietic tissues), adults (level 1) }\end{array}$ & 0,51 \\
\hline st19.075 & Лучевая терапия (уровень 1) / Radiation therapy (level 1) & 0,79 \\
\hline st19.078 & Лучевая терапия (уровень 4) / Radiation therapy (level 4) & 2,51 \\
\hline st19.081 & Лучевая терапия (уровень 7) / Radiation therapy (level 7) & 4,87 \\
\hline st19.084 & $\begin{array}{l}\text { Лучевая терапия в сочетании с лекарственной терапией (уровень 2) / } \\
\text { Radiation therapy in combination with drug therapy (level 2) }\end{array}$ & 3,78 \\
\hline st19.085 & $\begin{array}{l}\text { Лучевая терапия в сочетании с лекарственной терапией (уровень 3) / } \\
\text { Radiation therapy in combination with drug therapy (level 3) }\end{array}$ & 4,37 \\
\hline st19.088 & $\begin{array}{l}\text { Лучевая терапия в сочетании с лекарственной терапией (уровень 6) / } \\
\text { Radiation therapy in combination with drug therapy (level 6) }\end{array}$ & 9,49 \\
\hline st19.089 & $\begin{array}{l}\text { Лучевая терапия в сочетании с лекарственной терапией (уровень 7) / } \\
\text { Radiation therapy in combination with drug therapy (level 7) }\end{array}$ & 16,32 \\
\hline
\end{tabular}

$$
C_{\text {дс } / \text { Кс }}=N_{\text {дс } / \text { Кс }} \times \mathrm{K}_{\text {дС } / \text { Кс }} \times \mathrm{K}_{\text {дС } / \text { Кс }}
$$

где $\mathrm{C}_{\text {дс/Кс }}$ - средняя стоимость законченного случая лечения, включенного в КСГ, в условиях ДС/КС за счет средств ОМС; $N_{\text {дс/кс }}-$ средний норматив финансовых затрат на 1 случай лечения в условиях ДС/КС за счет средств ОМС; КП дс/Кс - коэффициент приведения при оплате специализированной медицинской помощи, принимающий значения не ниже 65\% и 60\% от значения норматива финансовых затрат на 1 случай госпитализации в условиях ДС и КС соответственно; КЗ дс/кс - коэффициент затратоемкости КСГ, к которой отнесен данный случай лечения.

В ходе проведения фармакоэкономического исследования условно было принято, что резекция, радиочастотная абляция, трансартериальная химиоэмболизация в 100\% случаев проводятся пациентам в КС. Также было принято допущение, что таргетную и лучевую терапию 50\% больных получают в ДС, а остальные 50\% - в КС (табл. 7).

Необходимо отметить, что в качестве стоимости проведения трансплантации печени была взята установленная в ПГГ средняя стоимость высокотехнологичной медицинской помощи (ВМП) № 61, которая составила 1257557 руб. ${ }^{6}$

\footnotetext{
${ }^{6}$ Постановление Правительства РФ от 28.12.2020 № 2299 «О Программе государственных гарантий бесплатного оказания гражданам медицинской помощи на 2021 год и на плановый период 2022 и 2023 годов».
}

Таблица 7

\section{Вероятность лечения пациента после установления диагноза гепатоцеллюлярного рака в дневном и круглосуточном стационарах, \%}

Table 7

Possibility of treating a patient after verification of hepatocellular carcinoma diagnosis in day-time and all-day inpatient facilities, \%

\begin{tabular}{l|c|c}
\hline \multicolumn{1}{c|}{$\begin{array}{c}\text { Вид лечения / } \\
\text { Type of treatment }\end{array}$} & $\begin{array}{c}\text { Дневной } \\
\text { стационар / } \\
\text { Day-time } \\
\text { facility }\end{array}$ & $\begin{array}{c}\text { Круглосуточный } \\
\text { стационар / } \\
\text { All-day facility }\end{array}$ \\
\hline $\begin{array}{l}\text { Резекция / Resection } \\
\text { Радиочастотная абляция / } \\
\text { Radiofrequenсу аblation } \\
\text { Tрансартериальная } \\
\text { химиоэмболизация / }\end{array}$ & 0 & 100 \\
$\begin{array}{l}\text { Transarterial } \\
\text { сhemoembolization } \\
\text { Tapгетная терапия / }\end{array}$ & 0 & 100 \\
$\begin{array}{l}\text { Targeted therapy } \\
\text { Лучевая терапия / } \\
\text { Radiation therapy }\end{array}$ & 50 & 50 \\
\hline
\end{tabular}

Средняя стоимость лечения 1 пациента по результатам диагностики ГЦР была рассчитана по следующей формуле:

$$
\begin{gathered}
C_{\text {леч }}=\sum_{i=1}^{n}\left(\mathrm{C}_{\text {дс } i} \times \text { Ддс } i+\mathrm{C}_{\mathrm{KC} i} \times \text { Д }_{\mathrm{KC} i}\right) \times P_{i}+ \\
+\mathrm{C}_{\mathrm{BM} \Pi} \times P_{\mathrm{T}}
\end{gathered}
$$


где $\mathrm{C}_{\text {дсі }}$ - средняя стоимость законченного случая i-го лечения, включенного в КСГ, в условиях ДС за счет средств ОМС (руб.); Ддсі - доля пациентов, которым было проведено $i$-е лечение в условиях ДС (\%); $\mathrm{C}_{\mathrm{KC} i}$ - средняя стоимость законченного случая $i$-го лечения, включенного в КСГ, в условиях КС за счет средств ОМС (руб.); ДКСi доля пациентов, которым было проведено $i$-е лечение в условиях КС (\%); $P_{i}$ - вероятность проведения $i$-го лечения после постановки диагноза ГЦР; $\mathrm{C}_{\mathrm{BM}}$ - средний норматив финансовых затрат на единицу объема специализированной медицинской помощи (стоимость трансплантации печени, ВМП № 61) (руб.); $P_{\mathrm{T}}$ - вероятность проведения трансплантации печени после постановки диагноза ГЦР.

Таким образом, итоговая стоимость исследуемых схем диагностики была рассчитана по формуле:

$$
\mathrm{C}_{\text {итог }}=\mathrm{C}_{\mathrm{KT}}+\mathrm{C}_{\text {МРКС }}+\mathrm{C}_{\text {леч}} \text {, }
$$

где $\mathrm{C}_{\mathrm{KT}}$ - стоимость КТ с контрастным средством

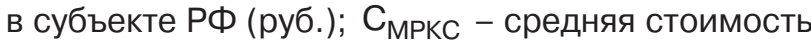
MPКC (руб.); $\mathrm{C}_{\text {леч }}$ - средняя стоимость лечения 1 пациента по результатам диагностики ГЦР (руб.).

\section{Анализ «затраты-эффективность»}

Завершающим этапом фармакоэкономического исследования было проведение анализа «затраты-эффективность». Результаты данного анализа представлены в виде коэффициента «затраты-эффективность» (cost-effectiveness ratio, (ER), демонстрирующего, какое количество денежных средств необходимо на 1 год жизни пациента, и инкрементального коэффициента «затратыэффективность» (incremental cost-effectiveness ratio, ICER), демонстрирующего, какое количество дополнительных денежных средств необходимо на 1 дополнительный год жизни пациента.

Для расчета показателей были использованы следующие формулы ${ }^{7}$ :

$$
\mathrm{CER}=\frac{\text { Cost }}{\mathrm{Ef}},
$$

где CER - коэффициент «затраты-эффективность»; Cost - стоимость медицинской технологии (руб.); показатель эффективности медицинской технологии (медиана выживаемости) (лет);

$$
\text { ICER }=\frac{\text { Cost }_{1}-\text { Cost }_{2}}{\mathrm{Ef}_{1}-\mathrm{Ef}_{2}},
$$

где ICER - инкрементальный показатель «затраты-эффективность»; Cost $_{1}$ - стоимость ана-

\footnotetext{
7 Приказ ФГБУ «ЦККЭМП» Минздрава России от 29.12.2018 №242-од «Методические рекомендации по проведению сравнительной клинико-экономической оценки лекарственного препарата».
}

лизируемой медицинской технологии (руб.); Cost $_{2}$ - стоимость сравниваемой медицинской технологии (руб.); $\mathrm{Ef}_{1}$ - показатель эффективности анализируемой медицинской технологии (медиана выживаемости) (лет); $\mathrm{Ef}_{2}$ - показатель эффективности сравниваемой медицинской технологии (медиана выживаемости) (лет).

\section{Результаты}

\section{Анализ эффективности}

Анализ эффективности показал, что смертность от всех причин у пациентов с ГЦР, диагностированным с помощью КТ, КT + МPT с Gd и КT + MPT с гадоксетовой кислотой, составила 36,3, 21,7 и 15,2 случая смерти на 100 человеко-лет соответственно. При этом медиана выживаемости составила 1,06 (95\% ДИ 1,01-1,11), 2,51 (95\% ДИ 2,35$2,70)$ и 4,80 (95\% ДИ 4,63-5,01) лет соответственно ( $<<0,001)$ (табл. 8) [23]. Таким образом, применение схемы диагностики КТ + МРТ с гадоксетовой кислотой позволило достичь почти у 50\% пациентов практически 5-летней выживаемости (4,8 года), что на 2,3 года (48\%) больше в сравнении с применением КT + MPT с Gd и на 3,7 года (78\%) больше в сравнении с КТ. В связи с этим КТ + МРТ с гадоксетовой кислотой показали себя как наиболее эффективный комплекс.

\section{Анализ затрат}

В результате расчета затрат было выявлено, что итоговая стоимость КТ + МРТ с гадоксетовой кислотой составляет 71338 руб., тогда как стоимость КТ и КT + МPT с Gd - 49332 руб. и 63486 руб. соответственно (табл. 9). Таким образом, анализ затрат показал, что применение схемы КТ + МРТ с гадоксетовой кислотой при диагностике ГЦР может привести к увеличению затрат на 7852 руб. по сравнению с КT + MPT с Gd и на 22006 руб. по сравнению с КТ.

\section{Анализ «затраты-эффективность»}

Необходимость проведения анализа «затратыэффективность» была обусловлена одновременным наличием двух факторов:

1) медиана выживаемости после проведения комплекса исследований КТ + МРТ с гадоксетовой кислотой статистически значимо выше медианы выживаемости после проведения КТ и КТ + MPT с Gd;

2) метод исследования КТ + МРТ с гадоксетовой кислотой характеризуется большими затратами, чем применение других диагностических процедур сравнения.

По результатам анализа «затраты-эффективность», схема КТ + МРТ с гадоксетовой кислотой при диагностике ГЦР является доминантной, т.к. она более эффективна и требует меньших 
Эффективность методов диагностики гепатоцеллюлярного рака [23]

Efficacy of diagnostic methods for hepatocellular carcinoma [23]

\begin{tabular}{|c|c|c|}
\hline $\begin{array}{c}\text { Метод диагностики / } \\
\text { Diagnostic method }\end{array}$ & $\begin{array}{l}\text { Медиана выживаемости } \\
\text { (95\% ДИ, p < 0,001), лет / } \\
\quad \text { Median survival rate } \\
(95 \% \mathrm{Cl}, \mathrm{p}<0,001) \text {, years }\end{array}$ & $\begin{array}{c}\text { Разница в медиане выживаемости в сравнении } \\
\text { с КТ + МРТ с гадоксетовой кислотой, лет* / } \\
\text { Difference in median survival rate compared } \\
\text { to CT + MRI with gadoxetoic acid, years* }\end{array}$ \\
\hline $\mathrm{KT} / \mathrm{CT}$ & $1,06(1,01-1,11)$ & 3,7 \\
\hline $\mathrm{KT}+\mathrm{MPT}$ c Gd / CT + MRI with GBCA & $2,51(2,35-2,70)$ & 2,3 \\
\hline $\begin{array}{l}\text { КТ + MPT с гадоксетовой кислотой / } \\
\text { СТ + MRI with gadoxetic acid }\end{array}$ & $4,80(4,63-5,01)$ & - \\
\hline
\end{tabular}

${ }^{*} p<0,001$.

Таблица 9

Итоговые затраты на 1 пациента при диагностике гепатоцеллюлярного рака

Table 9

Total costs per 1 patient for hepatocellular carcinoma diagnosis

\begin{tabular}{|c|c|c|c|c|}
\hline $\begin{array}{l}\text { Метод диагностики / } \\
\text { Diagnostic method }\end{array}$ & $\begin{array}{l}\text { Стоимость КТ, руб. / } \\
\text { Cost of CT, rub. }\end{array}$ & $\begin{array}{l}\text { Стоимость МРКС, руб./ } \\
\text { Cost of GBCA, rub. }\end{array}$ & $\begin{array}{l}\text { Стоимость лечения, руб. / } \\
\text { Cost of treatment, rub. }\end{array}$ & $\begin{array}{c}\text { Итоговая стоимость, руб. } \\
\text { Total cost, rub. }\end{array}$ \\
\hline $\mathrm{KT} / \mathrm{CT}$ & 7027 & - & 42305 & 49332 \\
\hline $\begin{array}{l}\mathrm{KT}+\mathrm{MPT} \text { c Gd / CT + } \\
+ \text { MRI with GBCA }\end{array}$ & 7027 & 2118 & 54342 & 63486 \\
\hline $\begin{array}{l}\text { КT + МРТ } \\
\text { с гадоксетовой } \\
\text { кислотой / СТ + MRI } \\
\text { with gadoxetic acid }\end{array}$ & 7027 & 7840 & 56471 & 71338 \\
\hline
\end{tabular}

Примечание. МРКС - магнитно-резонансное контрастное средство.

Note. GBCA - gadolinium-based contrast agent.

Таблица 10

Результаты анализа «затраты-эффективность»

Table 10

Results of cost-effectiveness analysis

\begin{tabular}{|c|c|c|c|}
\hline $\begin{array}{l}\text { Метод диагностики / } \\
\text { Diagnostic method }\end{array}$ & $\begin{array}{c}\text { Медиана выживаемости, } \\
\text { лет / Median survival rate, } \\
\text { years }\end{array}$ & $\begin{array}{c}\text { Итоговая стоимость, руб. / } \\
\text { Total cost, rub. }\end{array}$ & $\begin{array}{c}\text { Коэффициент «затраты- } \\
\text { эффективность» / } \\
\text { Cost-effectiveness ratio }\end{array}$ \\
\hline $\mathrm{KT} / \mathrm{CT}$ & 1,1 & 49332 & 46540 \\
\hline $\mathrm{KT}+\mathrm{MPT}$ c Gd / CT + MRI with GBCA & 2,5 & 63486 & 25293 \\
\hline $\begin{array}{l}\text { КT + MPT с гадоксетовой кислотой / } \\
\text { CT + MRI with gadoxetic acid }\end{array}$ & 4,8 & 71338 & 14862 \\
\hline
\end{tabular}

затрат на достижение 1 года жизни в сравнении с KT + MPT с Gd, а также в сравнении с КT (табл. 10).

При расчете инкрементальных показателей «затраты-эффективность» были получены следующие результаты:
- если пациенту вместо КT + MPT с Gd провести более эффективную диагностику КТ + МРТ с гадоксетовой кислотой, применение которой может позволить увеличить общую выживаемость на 2,3 года, то затраты на каждый дополнительный год жизни составят всего 3429 руб.; 
Incremental cost-effectiveness ratios calculated for hepatocellular carcinoma diagnostic methods

\begin{tabular}{|c|c|c|c|}
\hline $\begin{array}{l}\text { Методы диагностики / } \\
\text { Diagnostic method }\end{array}$ & $\begin{array}{c}\text { Разница } \\
\text { в эффективности, } \\
\text { лет жизни / } \\
\text { Difference } \\
\text { in efficiency, } \\
\text { life years }\end{array}$ & $\begin{array}{c}\text { Разница } \\
\text { в итоговой } \\
\text { стоимости, руб./ } \\
\text { Difference in total } \\
\text { cost, rub. }\end{array}$ & $\begin{array}{c}\text { Инкрементальный } \\
\text { показатель затраты- } \\
\text { эффективность / } \\
\text { Incremental } \\
\text { cost-effectiveness } \\
\text { ratio }\end{array}$ \\
\hline $\begin{array}{l}\text { KT + MPT с гадоксетовой кислотой против КT + MPT c Gd / } \\
\text { MRI with gadoxetoic acid vs. CT + MRI with GBCA }\end{array}$ & 2,3 & 7852 & 3429 \\
\hline $\begin{array}{l}\text { KT + MPT с гадоксетовой кислотой против КT / } \\
\text { MRI with gadoxetoic acid vs.CT }\end{array}$ & 3,7 & 22006 & 5884 \\
\hline KT + MPT c Gd против KT / CT + MRI with GBCA vs. CT & 1,4 & 14154 & 9762 \\
\hline
\end{tabular}

- если пациенту вместо КТ выполнить диагностику ГЦР при помощи КТ + МРТ с гадоксетовой кислотой, то медиана выживаемости может увеличиться на 3,7 года, а затраты на каждый дополнительный год жизни составят 5884 руб.;

- если пациенту вместо КТ провести КТ + МРТ c Gd, это приведет к увеличению медианы выживаемости на 1,4 года, при этом стоимость дополнительного года жизни составит 9762 руб.

Таким образом, схема диагностики КТ + МРТ с гадоксетовой кислотой характеризуется самым низким значением инкрементального показателя «затраты-эффективность», т.е. требует меньше дополнительных затрат на достижение 1 дополнительного года жизни (табл. 11).

\section{Ограничения исследования}

В основу выполненных расчетов экономической эффективности легли результаты ретроспективного когортного исследования T.W. Kang et al. (2020 г.), проведенного среди пациентов в Южной Kopeе. В связи с этим при интерпретации результатов нашего фармакоэномического анализа следует учитывать все ограничения указанного исследования.

Факторы риска развития ГЦР, структура здравоохранения и подходы к лечению ГЦР в Южной Корее могут отличаться от таковых в Российской Федерации. Например, диагностическая эффективность МРТ с гадоксетовой кислотой может быть различной у пациентов с неалкогольным циррозом печени, связанным со стеатогепатитом и другими фоновыми заболеваниями печени. Также на выживаемость при ГЦР могут влиять следующие факторы: наличие и степень нарушения функции печени, специализация и оснащение клиники, в которой лечится или наблюдается пациент, используемые методы лечения (в Южной Корее в основном проводят резекцию или радиочастотную абляцию, а не пересадку печени).

\section{Заключение}

Результаты проведенного фармакоэкономического исследования показали, что схема КТ + MPT с гадоксетовой кислотой является наиболее эффективным комплексом исследований для диагностики ГЦР, поскольку ее применение позволило достичь почти у 50\% пациентов практически 5-летней выживаемости (4,8 года), что статистически значимо выше, чем при использовании схем КТ + МРТ с внеклеточными гадолинийсодержащими МРКС (2,3 года) и КТ (3,7 года).

Схема обследования с гадоксетовой кислотой является доминантной для диагностики ГЦР, поскольку характеризуется статистически значимо более высокой медианой выживаемости, а также наименьшим количеством затрат на достижение 1 года жизни (14 862 руб.) по сравнению с КТ + МРТ с внеклеточными гадолинийсодержащими МРКС (25 293 руб.) или КТ (46 540 руб.). Кроме того, ее применение связано с меньшими дополнительными затратами на достижение 1 дополнительного года жизни.

Полученные в ходе исследования результаты свидетельствуют о том, что использование МРТ с гепатоспецифическим МРКС - гадоксетовой кислотой в дополнение к КТ с контрастированием является эффективным и экономически выгодным способом ранней диагностики ГЦР, что в свою очередь оказывает влияние на повышение резектабельности опухолей печени и общую выживаемость пациентов. 


\section{Литература [References]}

1. Coleman WB. Mechanisms of human hepatocarcinogenesis. Curr Mol Med. 2003; 3(6): 573-88. https://doi.org/10.2174/1566524033479546.

2. Lalazar G, Sanford MS. Fibrolamellar carcinoma: recent advances and unresolved questions on the molecular mechanisms. Semin Liver Dis. 2018; 38(1): 51-9. https://doi.org/10.1055/s-0037-1621710.

3. European Association for the Study of the Liver. EASL clinical practice guidelines: management of hepatocellular carcinoma. J Hepatol. 2018; 69(1): 182-236. https://doi.org/10.1016/j.jhep.2018.03.019.

4. Omata M, Cheng AL, Kokudo N, et al. Asia-Pacific clinical practice guidelines on the management of hepatocellular carcinoma: a 2017 update. Hepatol Int. 2017; 11(4): 317-70. https://doi.org/10.1007/s12072-017-9799-9.

5. Бредер В.В., Балахнин П.В., Виршке Э.Р. и др. Практические рекомендации по лекарственному лечению больных гепатоцеллюлярным раком. Злокачественные опухоли. 2021; 10(3s2-1): 450-69.

https://doi.org/10.18027/2224-5057-2020-10-3s2-25. [Breder VV, Balakhnin PV, Virshke ER, et al. Practical recommendations for drug treatment of patients with hepatocellular carcinoma. Malignant Tumors. 2021; 10(3s2-1): 450-69. https://doi.org/10.18027/2224-5057-2020-10-3s2-25 (in Russ.).]

6. Chagas AL, Kikuchi L, Herman P, et al. Clinical and pathological evaluation of fibrolamellar hepatocellular carcinoma: a single center study of 21 cases. Clinics (Sao Paulo). 2015; 70(3): 207-13. https://doi.org/10.6061/clinics/2015(03)10.

7. Jhaveri K, Cleary S, Audet P, et al. Consensus statements from a multidisciplinary expert panel on the utilization and application of a liver-specific MRI contrast agent (gadoxetic acid). Am J Roentgenol. 2015; 204(3): 498-509. https://doi.org/10.2214/AJR.13.12399.

8. Kojiro M. 'Nodule-in-nodule' appearance in hepatocellular carcinoma: its significance as a morphologic marker of dedifferentiation. Intervirology. 2004; 47(3-5): 179-83. https://doi.org/10.1159/000078470.

9. An C, Rhee H, Han K, et al. Added value of smooth hypointense rim in the hepatobiliary phase of gadoxetic acid-enhanced MRI in identifying tumour capsule and diagnosing hepatocellular carcinoma. Eur Radiol. 2017; 27(6): 2610-18. https://doi.org/10.1007/s00330-016-4634-6.

10. Tang A. Using MRI to assess microvascular invasion in hepatocellular carcinoma. Radiology. 2020; 297(3): 582-3. https://doi.org/10.1148/radiol.2020203376.

11. Zech CJ, Ba-Ssalamah A, Berg T, et al. Consensus report from the 8th international forum for liver magnetic resonance imaging. Eur Radiol. 2020; 30(1): 370-82. https://doi.org/10.1007/s00330-019-06369-4.

12. Marrero JA, Kulik LM, Sirlin CB, et al. Diagnosis, staging, and management of hepatocellular carcinoma: 2018 Practice Guidance by the American Association for the Study of Liver Diseases. Hepatology. 2018; 68(2): 723-50. https://doi.org/10.1002/hep.29913.
13. 2018 Korean Liver Cancer Association - National Cancer Center Korea Practice Guidelines for the management of hepatocellular carcinoma. Gut Liver. 2019; 13(3): 227-99. https://doi.org/10.5009/gnl19024.

14. Benson AB, D’Angelica MI, Abbott DE, et al. Guidelines insights: hepatobiliary cancers, version 2.2019. J Natl Compr Canc Netw. 2019; 17(4): 302-10. https://doi.org/10.6004/jnccn.2019.0019.

15. Vogel A, Cervantes A, Chau I, et al. Hepatocellular carcinoma: ESMO Clinical Practice Guidelines for diagnosis, treatment and follow-up. Ann Oncol. 2018; 29(Suppl. 4): 238-55. https://doi.org/10.1093/annonc/mdy308.

16. Kudo M, Matsul O, Izumi N, et al. JSH consensus-based clinical practice guidelines for the management of hepatocellular carcinoma: 2014 update by the Liver Cancer Study Group of Japan. Liver Cancer. 2014; 3(3-4): 458-68. https://doi.org/10.1159/000343875.

17. European Association for the Study of the Liver. EASL Clinical Practice Guidelines on the management of benign liver tumors. J Hepatol. 2016; 65(2): 386-98. https://doi.org/10.1016/j.jhep.2016.04.001.

18. Клинические рекомендации «Рак печени (гепатоцеллюлярный)», 2020. URL: https://old.oncology-association.ru/files/clinicalguidelines-2020/rak_pecheni.pdf (дата обращения 18.08.2021).

[Clinical guidelines "Hepatocellular carcinoma”. Available at: https://old.oncology-association.ru/files/clinicalguidelines-2020/rak_pecheni.pdf (accessed August 18, 2021) (in Russ.).]

19. Van Cutsem E, Cervantes A, Adam R, et al. ESMO consensus guidelines for the management of patients with metastatic colorectal cancer. Ann Oncol. 2016; 27(8): 1386-422. https://doi.org/10.1093/annonc/mdw235.

20. Zech C, Grazioli L, Jonas E, et al. Health-economic evaluation of three imaging strategies in patients with suspected colorectal liver metastases: Gd-EOB-DTPA-enhanced MRI vs. extracellular contrast media-enhanced MRI and 3-phase MDCT in Germany, Italy and Sweden. Eur Radiol. 2009; 19(Suppl. 3): 753-63. https://doi.org/10.1007/s00330-009-1432-4.

21. Lee JM, Kim MJ, Phongkitkarun S, et al. Health economic evaluation of Gd-EOB-DTPA MRI vs ECCM-MRI and multidetector computed tomography in patients with suspected hepatocellular carcinoma in Thailand and South Korea.J Med Econ. 2016; 19(6): 759-68. https://doi.org/10.3111/13696998.2016.1171230.

22. Nishie A, Goshima S, Haradome H, et al. Cost-effectivenss of EOB-MRI for hepatocellular carcinoma in Japan. Clin Ther. 2017; 39(3): 738-50.e4. https://doi.org/10.1016/j.clinthera.2017.03.006.

23. Kang TW, Kong SY, Kang D, et al. Use of gadoxetic acidenhanced liver MRI and mortality in more than 30000 patients with hepatocellular carcinoma: a nationwide analysis. Radiology. 2020; 295(1): 114-24. https://doi.org/10.1148/radiol.2020190639. 